“(C) 2015 IEEE. Personal use of this material is permitted. Permission from IEEE must be obtained for all other uses, in any current or future media, including reprinting/republishing this material for advertising or promotional purposes, creating new collective works, for resale or redistribution to servers or lists, or reuse of any copyrighted component of this work in other works." 


\title{
A Geometrical Sink-Based Cooperative Coverage Hole Recovery Strategy for WSNs
}

\author{
Ali Rafiei, Yashar Maali,Mehran Abolhasan, Daniel Franklin \\ Faculty of Engineering and Information Technology \\ University of Technology, Sydney, Sydney, Australia \\ Email: ali.rafiei@ieee.org, \{yashar.maali,mehran.abolhasan,daniel.franklin\}@uts.edu.au
}

\begin{abstract}
Unlike sporadic node failures, coverage holes emerging from multiple temporally-correlated node failures can severely affect quality of service in a network and put the integrity of entire wireless sensor networks at risk. Conventional topology control schemes addressing such undesirable topological changes have usually overlooked the status of participating nodes in the recovery process with respect to the deployed sink node(s) in the network. In this paper, a cooperative coverage hole recovery model is proposed which utilises the simple geometrical procedure of circle inversion. In this model, autonomous nodes consider their distances to the deployed sink node(s) in addition to their local status, while relocating towards the coverage holes. By defining suitable metrics, the performance of our proposed model performance is compared with a force-based approach.
\end{abstract}

\section{INTRODUCTION}

In order to deliver a reliable and robust Quality of Service in different applications, network faults' effects in wireless sensor networks (WSNs) should be systematically managed [1]. Amongst such faults, Coverage Holes (CHs), as the result of correlated node failures, can disturb QoS and even affect the network's integrity at larger scales [2], [3], [4]. Integrating mobility capabilities into sensor nodes and harnessing different mobility patterns [5], [6] enables the problem of $\mathrm{CHs}$ and other sudden dynamic change to network topology to be automatically corrected [4], [7], [8], [9]. Distributed node relocation algorithms [6], [10], [8] which can direct the movement of mobile nodes have emerged as an interesting approach to topology control (TC) in WSNs [11], enabling mitigation of the effects of random node failures and $\mathrm{CH}$ formation in unsupervised networks.

Using the simple geometrical process of circle inversion [12], we propose a sink-based coverage hole recovery model in which nodes modify their position with respect to not only their neighbouring nodes but also deployed sink nodes during the process of relocating towards $\mathrm{CHs}$. The modifications in the direction and magnitude of nodes' movements depend on their respective transmission ranges and distances to the sink nodes. The general idea of the proposed recovery model is shown in Fig. 1 for one coverage hole in the network.

The logic behind proposed model relates to the effect of sink nodes on their neighbours. Nodes in proximity to a network sink relay traffic for the surrounding region of the network in addition to their own data to the sink nodes. Hence, their energy supply will be exhausted faster than that of average nodes in the rest in the network, which may disproportionately

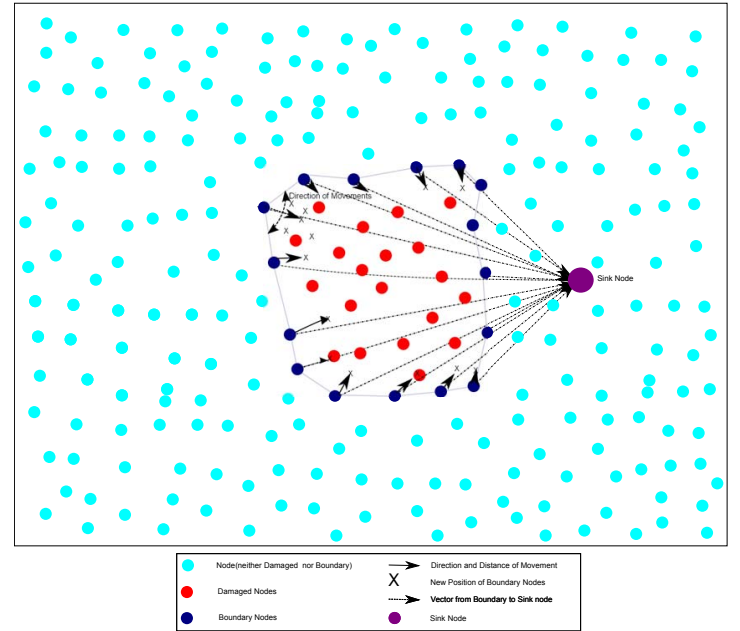

Fig. 1. Relocation of boundary nodes in sink-based $\mathrm{CH}$ recovery model

degrade the process of data collection from the sensor network [13]. Therefore, it would be desirable that participating nodes' distances to the deployed sink nodes be reduced during the course of $\mathrm{CH}$ recovery.

The proposed recovery model allows autonomous nodes to detect the presence of $\mathrm{CHs}$ and swiftly relocate towards the damaged areas based on their knowledge of the extent of the $\mathrm{CHs}$ and the number and location of neighbours and deployed sink nodes. This local decision-making process among autonomous nodes results in emergent cooperation [14]. The proposed algorithm is suitable for delay-sensitive applications and security-sensitive applications in which the need to avoid location disclusre requires that the amount of information exchanged between nodes should be minimised. The model is well-suited for networks in harsh and hostile environments with no or limited centralised control.

In this paper, performance of the proposed model is compared with that of a force-based approach (DSSA) [10] and two Voronoi-based relocation algorithms [7].

In Section II, a summary of the most significant related work is presented. In Section III, the proposed model and assumptions are introduced. Performance metrics and results discussed in sections IV and V, respectively. Finally, VI concludes the paper and summarises the key findings. 


\section{RELATED WORK AND MotiVATION}

To recover from or reduce the effects of dynamicallyformed coverage holes of different scales [2], [3], [4], a wide spectrum of topology control schemes has been devised [15], [11]. By providing a degree of control over the coverage and connectivity of networks, topology control schemes using distributed node relocation algorithms are able to maintain or recover network integrity in networks subject to dynamic topological perturbation [6], [7], [16], [9], [4], [10], [17]. Node relocation algorithms can be broadly classified into a number of major categories [4], including Force-based algorithms in which nodes mutually exert virtual repulsive and attractive forces in the radial [10] or angular [16] directions, voronoibased approaches in which movement is based on Voronoi cells formed by the nodes[7], and flip-based and cell-based relocation algorithms, in which nodes are flipped to adjacent cells in a manner depending on cell resolution, node density and decisions of cells' elected head nodes [9].

During the recovery process, the scale of the coverage holes and the proportion of participating nodes in the network has a significant impact on the speed and efficacy of relocation algorithms [18]. Especially, the particular spatial distribution of recover-participating nodes with respect to the location of network sink nodes can significantly impact the useable lifespan of the network under conditions of constrained power availability, since these nodes will expend a greater fraction of their energy budget on both movement and relaying traffic for the rest of the network. Therefore, by exploiting the autonomous nodes' knowledge of sink nodes locations and using simple geometrical properties and procedures, it will be possible for node relocation algorithms to avoid excess node energy depletion in the vicinity of sink nodes while still reaching the primary design goals (i.e., repair and/or maintenance coverage of damaged areas).

\section{Methods And Assumptions}

Homogeneous sensor nodes with communication range modelled with a unit disk graph (UDG) are randomly deployed in $2 \mathrm{D}$ rectangular region of $\left[x_{\min }, x_{\max }\right] \times\left[y_{\min }, y_{\max }\right]$ with a 2D uniform random spatial distribution. For simplicity, nodes transmission and sensing ranges of $R_{c}$ and $R_{s}$ are considered to be equal. Therefore, two nodes are bidirectionally connected if they are located within each others' communication ranges. Nodes are aware of their own position via GPS receivers or some other localisation methods [19].

Sink nodes $S_{s}(k) \in\left\{S_{s}(1), \cdots, S_{s}\left(N_{s}\right)\right\}$ are deployed at locations $\left(x_{s_{k}}, y_{s_{k}}\right)$. Here, it is assumed that a single sink node is deployed in the entire network; however the model drived in this Section is applicable for networks with more than one sink nodes. It is assumed that all nodes are aware of the deployed sink node's location.

\section{A. Coverage Holes and Node Types}

Coverage holes (CHs) result from temporally correlated node failures and can be modelled by a union of circles of radii $R_{h(l)}$ with centres located at $\left(x_{h_{l}}, y_{h_{l}}\right)$ for $l \in\left\{1, \cdots, N_{E}\right\}$

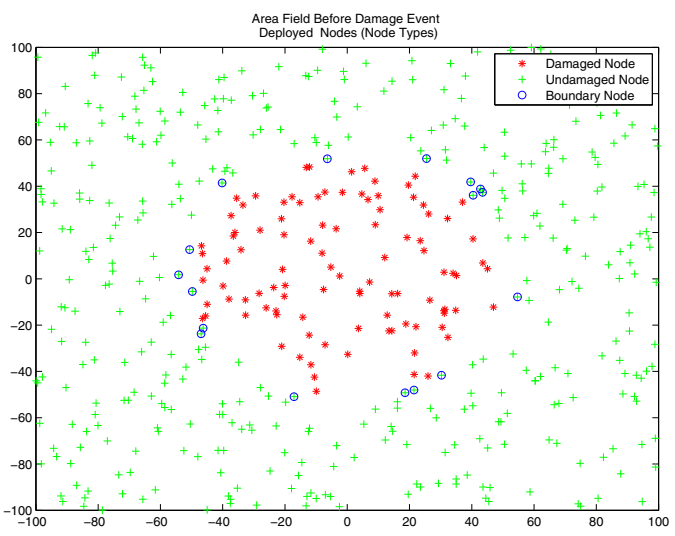

Fig. 2. Coverage hole, $N=500$ nodes, and $R_{c}=15(\mathrm{~m})$ [18]

where $N_{E}$ is the number of damage events. Nodes can be classified into undamaged nodes (U-nodes) and damaged nodes ( $D$-nodes), where the former reside outside and the latter inside the coverage hole. (Fig. 1,2). Undamaged nodes which detect the presence of a $\mathrm{CH}$ within their range are defined as boundary nodes (B-nodes). Fig. 2 shows an example of a coverage hole with $R_{h}=15 \mathrm{~m}$ and centre $\left(x_{h_{l}}, y_{h_{l}}\right)$ at $(0,0)$ in a network of $N=500$ nodes.

It is assumed that B-nodes can detect the damage event if at least one of their neighbours fails (i.e., is in the set of damaged nodes). Those undamaged nodes which are not B-nodes are considered to be normal nodes ( $N$-nodes). It is assumed that the sequence of coverage holes occurs with random radius and at uniformly randomly distributed locations within area of network deployment. Autonomous nodes commence the recovery procedure following detection of a damage event. It is assumed that B-nodes update their undamaged and damaged neighbours status after the movement and before the occurrence of further coverage hole-creating events. Therefore, B-nodes move based on status of their current neighbours at the time of event.

Fig. 3 schematically depicts a B-node together with each of its undamaged (blue circle) and damaged (red circle with cross) neighbour nodes within its communication range.

\section{B. Inter-Node Communication Protocol}

Prior to the formation of a coverage hole, nodes are aware of their neighbours' degrees of connectivity and position in addition to the location of deployed sink nodes.

It is assumed that at time of the damage event, nodes do not broadcast any additional information to their neighbours due to security considerations. Therefore, each B-node should autonomously decide on the its movement vector based on its knowledge at the time of the damage event. After a damage event, B-nodes are not able to to determine whether or not their undamaged neighbours are also B-nodes. Fig. 3 shows a B-node and its damaged and undamaged neighbours. It should be noted that a B-node can detect the occurrence of successive 


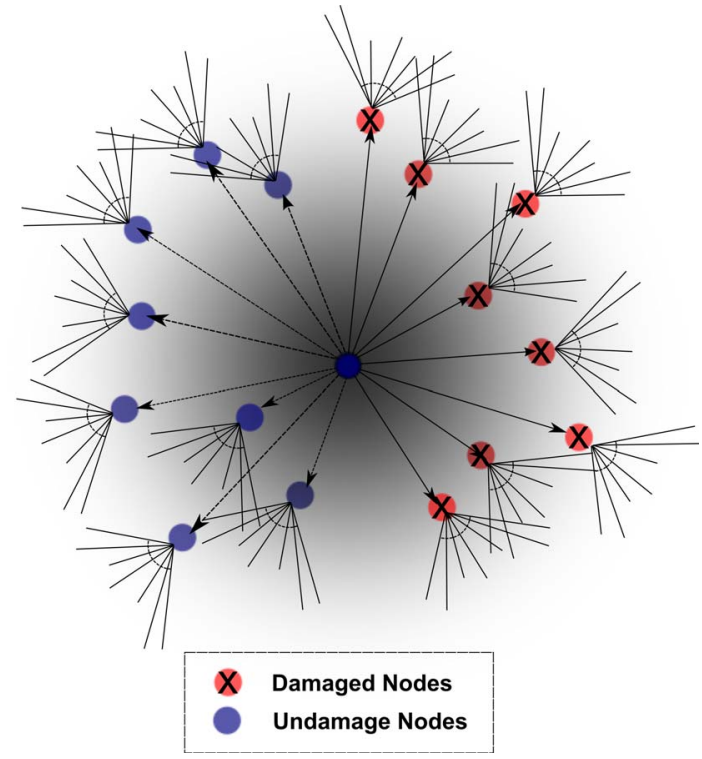

Fig. 3. B-node and its undamaged and damaged neighbours

damage events as long as it loses at least one of its neighbours due to each damage event.

\section{Node Movement Decision}

Movements of autonomous boundary nodes are determined using the parameters listed below:

- B-node's Neighbours: Those undamaged and damaged neighbours with respective distances of $\vec{x}_{S_{b}(i)}^{S_{N u}(l, j)}$ and $\vec{x}_{S_{b}(i)}^{S_{N d}(l, j)}$ which have the highest degrees of connectivity of ${ }_{S_{b}(i)}^{S_{N u}(l, j)}$ and $d_{S_{b}(i)}^{S_{N d}(l, j)}$ are considered to be the best nodes on which to base the movement decision of the given B-node $S_{b}(i)$. This is because if these parameters are properly weighted (i.e., as in (9)), then the B-node will move in the direction which improves coverage for its furthest damaged neighbours of the highest degrees, while at the same time maintaining its connectivity with its undamaged neighbours to increase the chance of remaining connected to the rest of the network despite its autonomous relocation.

- Sink node's status: B-node $S_{b}(i)$ 's displacement vector to the deployed sink node $S_{k}, \vec{x}_{S_{b}(i)}^{S_{s}(k)}$ with respect to nodes' ranges are used to modify the relocation of nodes towards the coverage holes (refer to Section III-E).

- Coverage hole: the number, locations, and dimensions of the coverage holes affect nodes' relocations in the recovery process. The $\mathrm{CHs}^{\prime}$ distances to the sink nodes also affect the performance of recovery model.

\section{Centre of Mass of B-nodes' Neighbours}

The centre of mass of B-node $S_{b}(i)$ 's neighbouring damaged and undamaged nodes should be calculated such that neighbouring undamaged and damaged nodes with higher degrees should have a greater weight in the determination of

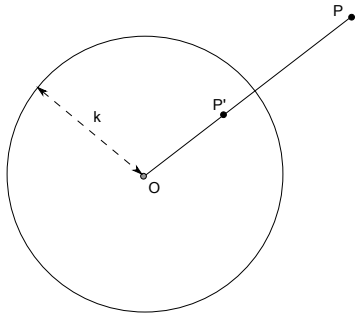

Fig. 4. Circle inversion

the boundary nodes' movement vectors. The idea of using the centre of mass of the B-nodes' (undamaged and damaged) neighbours is that each B-node moves as much as possible in the direction of damaged nodes with higher degrees of connectivity while maintaining its connection with network as far as possible. It is assumed that boundary nodes are aware of their neighbours' degrees before the occurrence of each damage event. Node degrees can be used to obtain the centre of mass of B-nodes' undamaged and damaged neighbouring nodes as in (1) and (2).

$$
\begin{aligned}
& \vec{M}_{s_{b}(i)}^{C M_{l} U}=\frac{\sum_{j=0}^{N u}\left(d_{S_{b}(i)}^{S_{N u}(l, j)} \cdot \vec{x}_{S_{b}(i)}^{S_{N u}(j, l)}\right)}{\sum_{j=0}^{N d} d_{S_{b}(i)}^{S_{N u}(l, j)}} \\
& \vec{M}_{s_{b}(i)}^{C M_{l} D}=\frac{\sum_{j=0}^{N d}\left(d_{S_{b}(i)}^{S_{N d}(l, j)} \cdot \vec{x}_{S_{b}(i)}^{S_{N d}(j, l)}\right)}{\sum_{j=0}^{N d} d_{S_{b}(i)}^{S_{N d}(l, j)}}
\end{aligned}
$$

Choosing proper combinations of undamaged and damaged centre of masses provides a logical criterion for the B-nodes' movement vectors. In the case that a B-node looses all of its undamaged and/or damaged neighbouring nodes, then its corresponding centres of mass are considered to be zero (null, i.e. not to be used).

$$
\begin{aligned}
& \vec{M}_{s_{b}(i)}^{C M_{l} U}=\overrightarrow{0}, \quad \text { if } N_{u}=0 \\
& \vec{M}_{s_{b}(i)}^{C M_{l} D}=\overrightarrow{0}, \quad \text { if } N_{d}=0
\end{aligned}
$$

\section{E. Effect of Sink Node}

The amount of movement in the direction of the deployed sink node is computed based on the idea of circle inversion[12] (Fig. 4).

As shown in Fig. 4, the reverse point $P^{\prime}$ of point $P$ can be found where $k^{2}$ is defined as the circle power in [12].

$$
\|O P\| \times\left\|O P^{\prime}\right\|=k^{2}
$$

Using (3), if $k=R_{c\left(S_{b}(i)\right)},\|O P\|=\| P_{\text {new }}\left(S_{b}(i)\right)-$ $P_{c u r}\left(S_{b}(i)\right) \|$, and $\left\|O P^{\prime}\right\|=\left\|\vec{x}_{S_{b}(i)}^{S_{s}(k)}\right\|$ where $P_{c u r}$ and 
$P_{\text {new }}$ are the current and new positions of boundary node $S_{b}(i)$ respectively before and after the damage event.

Hence, if

$$
\left\|P_{\text {new }}\left(S_{b}(i)\right)-P_{\text {cur }}\left(S_{b}(i)\right)\right\| \times\left\|\vec{x}_{S_{b}(i)}^{S_{s}(k)}\right\|=R_{S_{b}(i)}^{2}
$$

where $\left\|\vec{x}_{S_{b}(i)}^{S_{s}(k)}\right\|$ is distance of sink node $S_{s}(j)$ to the $S_{b}(i)$ and $R_{S_{b}(i)}$ is range of sensor node $S_{b}(i)$, then following cases can be considered:

1) $\left\|\vec{x}_{S_{b}(i)}^{S_{s}(k)}\right\|<R_{S_{b}(i)}$, as sink node $S_{s}(k)$ is already within the range of B-node $S_{b}(i)$. In this case, it is assumed that the movement of B-node $S_{b}(i)$ in the direction of sink node $S_{s}(k)$ is not required. So,

$$
\left\|P_{\text {new }}\left(S_{b}(i)\right)-P_{\text {cur }}\left(S_{b}(i)\right)\right\|=0,
$$

This is the case where sink node (point $P$ ) is within the range of B-node $S_{b(i)}$. In this case, moving B-node is devised not to move any further toward the sink node in which result less possible collision among the nodes and to prevent the increasing chance of the interference.

2) $\left\|\vec{x}_{S_{b}(i)}^{S_{s}(k)}\right\|>R_{S_{b}(i)}$, the amount of movement node $S_{b}(i)$ toward the sink node is obtained as

$$
\left\|P_{\text {new }}\left(S_{b}(i)\right)-P_{\text {cur }}\left(S_{b}(i)\right)\right\|=\frac{R_{S_{b}(i)}^{2}}{\left\|\vec{x}_{S_{b}(i)}^{S_{s}(k)}\right\|}
$$

3) if $\left\|\vec{x}_{S_{b}(i)}^{S_{s}(k)}\right\|=R_{S_{b}(i)}$, then the magnitude of the movement calculated using (6) is modified by a uniformly-distributed random factor $\rho_{\text {col }} \sim U[0,1]$ :

$$
\left\|P_{\text {new }}\left(S_{b}(i)\right)-P_{c u r}\left(S_{b}(i)\right)\right\|=\frac{R_{S_{b}(i)}^{2}}{\left\|\vec{x}_{S_{b}(i)}^{S_{s}(k)}\right\|} \cdot \rho_{c o l}
$$

The random factor $\rho_{\text {col }}$ reduces the chance of collision of nodes moving towards the deployed sink node.

Therefore, utilisation of the geometric procedure of circle inversion causes B-nodes to modify their movement vectors based on their distances to the sink nodes in addition to their communications range. B-nodes further from from the sink nodes move a smaller distance compared to B-nodes nearer to the sink, in order to reflect the nodes' relative importance to the network with respect to their proximity to the deployed sink nodes.

\section{F. Movement Toward CHs}

Suppose that the total number of neighbours of B-node $S_{b}(i)$ prior to the formation of $\mathrm{CH} l$ is $N_{S_{b}(i)}^{l}$. Let the number of undamaged and damaged neighbouring nodes of $S_{b}(i)$ after damage event $l$ to be $N_{S_{b}(i)}^{U_{l}}$ and $N_{S_{b}(i)}^{D_{l}}$, respectively. The damage ratio of B-node $S_{b}(i)$ due to the formation of coverage hole $l$ is defined as:

$$
\varepsilon_{S_{b}(i)}^{d_{l}}=\frac{N_{S_{b}(i)}^{D_{l}}}{N_{S_{b}(i)}^{U_{l}}+N_{S_{b}(i)}^{D_{l}}}
$$

Using the sigmoid function $\sigma(t)=\frac{1}{1+e^{-t}}, S_{b}(i)$ 's damage ratio can be used to determine the weighting between the damaged and undamaged nodes:

$$
\begin{aligned}
& \omega_{N_{S_{b}(i)}^{D_{l}}}=1-\sigma\left(\left\{\varepsilon_{S_{b}(i)}^{d_{l}}\right\}^{-1}\right) \\
& \omega_{N_{S_{b}(i)}^{U_{l}}}=1-\omega_{N_{S_{b}(i)}^{D_{l}}}
\end{aligned}
$$

where $\omega_{N_{S_{b}(i)}^{U_{l}}}$ and $\omega_{N_{S_{b}(i)}^{D_{l}}}$ are complementary weights used to combine the vectors obtained from $S_{b}(i)$ to the centres of mass of $S_{b}(i)$ 's undamaged and damaged neighbours due to coverage hole $l$.

\section{G. Proposed Movement Model}

Using the defined parameters, the following movement models are introduced. In all cases, nodes are constrained to remain within the boundaries $x_{\min }, x_{\max }, y_{\min }, y_{\max }$.

Simple Sink Movement (SSM), in which each moving Bnode $S_{b}(i)$ moves strictly towards the deployed sink node regardless of its status with respect to the coverage hole. Thus, depending on the location of damage event and the sink node, the recovery of coverage hole may not be primary goal of the movement algorithm. Using circle inversion, B-nodes move in the direction of the sink node. The magnitude of each B-node's movement depends on the node's own communication range and its distance to the given deployed sink node. So for each B-node, the movement $\vec{M}_{s_{b}(i)}^{s_{k}}$ can be defined as:

$$
\begin{aligned}
\vec{M}_{s_{b}(i)}^{s_{k}}= & P_{\text {new }}\left(S_{b}(i)\right)-P_{\text {cur }}\left(S_{b}(i)\right) \\
& \vec{M}_{s_{b}(i)}^{\text {Total }_{l}}=\vec{M}_{s_{b}(i)}^{s_{k}}
\end{aligned}
$$

In the case of multiple deployed sink nodes, movement of boundary nodes can be modified by considering the combined importance of the effect of those sink nodes as:

$$
\vec{M}_{s_{b}(i)}^{\text {Total }_{l}}=\sum_{k=1}^{N_{s}} \zeta_{k} \cdot \vec{M}_{s_{b}(i)}^{s_{k}}
$$

where $\zeta_{k}$ is the weight of importance for each sink node $k$ based on the conditions of the network.

If each B-node strictly moves towards the coverage hole regardless of the status of deployed sink nodes, the amount and directions of movement of nodes can be governed by the centres of mass of the B-nodes' undamaged and damaged neighbours as described in Section III-F. In this case, recovery of the coverage holes would be the primary objective of node movement rather than the consideration of the imporance of sink nodes. The movement of B-nodes is now given by:

$$
\vec{M}_{s_{b}(i)}^{C M_{l}}=f\left(\vec{M}_{s_{b}(i)}^{C M_{l} U}, \vec{M}_{s_{b}(i)}^{C M_{l} D}\right)
$$

where $\vec{M}_{s_{b}(i)}^{C M_{l} U}$ and $\vec{M}_{s_{b}(i)}^{C M_{l} U}$ are centres of mass of the B-node's undamaged and damaged neighbours, respectively, computed as described in Section III-F. The role of function 
$f$ is to tune the relative effect of the centres of masses based on the status of B-nodes and the $\mathrm{CH}$ as describe in Section III-F and (8).

$$
\vec{M}_{s_{b}(i)}^{\text {Total }_{l}}=\vec{M}_{s_{b}(i)}^{C M_{l}}
$$

Combined SS and CM Movement (CSSCMM): In this algorithm, the movement vector for each B-node is dependent on both the centres of mass of neighbouring nodes and the sink node location, with the weight $\lambda_{s} \in[0,1]$ defined as the sink move weight and $\lambda_{c m}=1-\lambda_{s}$ as the centres of mass weight. Using these weights, total movements are linear combinations of (10) and (12):

$$
\vec{M}_{s_{b}(i)}^{C m b_{l}}=\lambda_{s} \cdot \vec{M}_{s_{b}(i)}^{C M_{l}}+\lambda_{c m} \cdot \vec{M}_{s_{b}(i)}^{s_{k}}
$$

where $\vec{M}_{s_{b}(i)}^{C m b_{l}}$ is B-node $s_{b}(i)$ 's combined movement vector due to coverage hole $l$.

$$
\vec{M}_{s_{b}(i)}^{\text {Total }_{l}}=\vec{M}_{s_{b}(i)}^{C m b_{l}}
$$

Modified CSSCMM (M-CSSCMM): in this algorithm, the magnitudes of the B-nodes' movement vectors are tuned with regard to the status of B-nodes around the coverage hole and the sink node as defined in (16)-(19):

$$
\begin{aligned}
& \alpha_{\langle c m, s\rangle}= \\
& \left\{\begin{array}{cc}
\arccos \left[\frac{\vec{M}_{s_{b}(i)}^{C M_{l}} \cdot \vec{M}_{s_{b}(i)}^{s_{k}}}{\left\|\vec{M}_{s_{b}(i)}^{C M_{l}}\right\|\left\|\vec{M}_{s_{b}(i)}^{s_{k}}\right\|}\right], & \text { if }\left\|\vec{M}_{s_{b}(i)}^{C M_{l}}\right\|,\left\|\vec{M}_{s_{b}(i)}^{s_{k}}\right\| \neq 0 \\
0, & \text { Otherwise }
\end{array}\right.
\end{aligned}
$$

where $\alpha_{\langle c m, s\rangle}$ is the angle between movement vectors obtained from (10) and (12);

$$
\beta_{\langle c m, s\rangle}^{l}=\alpha_{\langle c m, s\rangle} \cdot\left(\frac{\theta_{\tau}}{\pi / 2}\right)^{-1}
$$

where $\theta_{\tau}$ is defined as the threshold angle and $\beta_{\langle c m, s\rangle}^{l}$ is defined as the modification angle.

$$
\gamma_{\langle c m, s\rangle}^{l}=\cos ^{2}\left(\beta_{\langle c m, s\rangle}^{l}\right)
$$

and the attenuated movement is obtained using (14).

$$
\vec{M}_{s_{b}(i)}^{\text {Total }_{l}}=\left\{\begin{array}{cc}
\gamma_{\langle c m, s\rangle}^{l} \cdot \vec{M}_{s_{b}(i)}^{C M_{l}}, & \text { if }\left(\beta_{\langle c m, s\rangle}^{l}<1\right), \\
0, & \text { Otherwise }
\end{array}\right.
$$

The idea of M-CSSCMM is that the flexiblitiy of B-nodes' movement can be configured according to the angle between the two movement vectors that each B-node forms with its neighbours' centres of mass and the deployed sink node. In (19), if $\gamma_{\langle c m, s\rangle}^{l}=1$, the movement of B-nodes is determined solely by the angle $\alpha_{\langle c m, s\rangle}$. This movement varient of $\mathrm{M}$ CSSCMM is denoted as (dM-CSSCMM). If the condition of $\beta_{\langle c m, s\rangle}^{l}<1$ is relaxed, the movement algorithm is denoted as $(a M-C S S C M M)$ in which only the amplitudes of movements are attenuated.

\section{H. Physical Movement}

To reduce physical collisions and unnecessary movements among autonomous nodes, before actual physical movement is allowed to commence, a threshold $\delta_{\tau} \in[0,1]$ is defined such that B-nodes compare a uniformly-distributed random variable $\nu_{S_{b}(i)}^{l} \sim U[0,1]$ with the given threshold. Whether B-nodes decide to actually trigger physical movement or not depends on the condition below:

$$
\vec{M}_{s_{b}(i)}^{P H Y_{l}}=\left\{\begin{array}{cc}
\gamma_{\langle c m, s\rangle}^{l} \cdot \vec{M}_{s_{b}(i)}^{C M_{l}}, & \text { if }\left(\nu_{s_{b}(i)}^{l} \geq \delta_{\tau}\right) \\
0, & \text { Otherwise }
\end{array}\right.
$$

\section{Performance Metrics}

The following performance metrics have been used for evaluating the performance of the proposed model.

1) Percentage of Coverage: The 2D rectangular deployment area $\left(\left[x_{\min }, x_{\max }\right] \times\left[y_{\min }, y_{\max }\right]\right)$ is divided into grid cells. Grid cells are covered by sensor nodes if their coordinates $z_{i}=\left(x_{i}, y_{i}\right)$ reside within the nodes' ranges. Percentage of 1-coverage is the percentage of grid cells covered by at least one sensor node out of the total number of grid cells in the deployed area.

2) B-Nodes to Sink Node Distances: In the recovery of consecutive random $\mathrm{CHs}$, it is desirable that the moving $\mathrm{B}$ nodes maintain and/or reduce their distances to the deployed sink nodes in the network. Thus, as the result of the proposed recovery model, the distances of the set of moving B-nodes to the deployed sink node(s) are expected to be monotonic decreasing while the selected B-nodes relocate towards the $\mathrm{CHs}$. The performance of movement algorithms are examined by cumulative mean of maximum, minimum and average distances of B-nodes to the deployed sink nodes in the course of recovery of consecutive random $\mathrm{CHs}$ in the network.

3) Sparsity of Cut: Spectral graph theory metrics provide a useful measure of network robustness and reliability. The clique is considered to be the most reliable network layout if a fraction of vertices are disconnected from the graph [20], [21]; therefore, the number of cliques is an indicator of the robustness of the network. Specifically, the sparsity of cut, which is a measure of the 'efficiency' of some specific partition of a network graph, can be used to measure network reliability of network with respect to the ideal clique.

Definition 1: [22], [21], Let $G=(V, E)$ be a graph and let $(S, V-S)$ be a partition of the vertices (a cut). Then the sparsity of the cut is

$$
\phi(S):=\frac{E(S, V-S)}{|E|} \cdot\left(\frac{|S| \cdot|V-S|}{|V|^{2} / 2}\right)^{-1}
$$

where $E(S, V-S)$ is the number of edges in $E$ that have one endpoint in $S$ and one end point in $V-S$.

Sparsity can be used as a measure of how the set of B-nodes are connected to the normal (undamaged, nonboundary) nodes. A higher sparsity cut requires a greater number of link disconnections to create a partitioned network. The performance of movement algorithms are compared by 


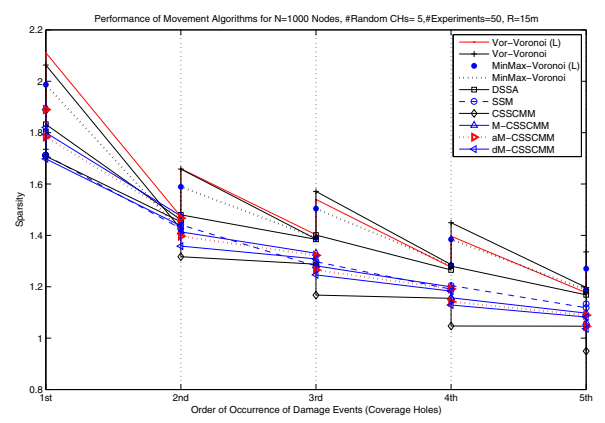

Fig. 5. Network sparsity

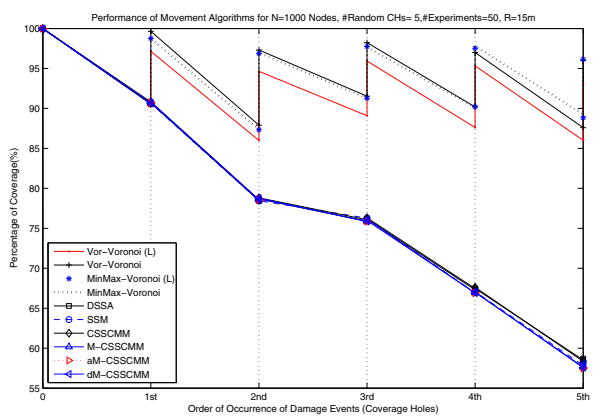

Fig. 6. Percentage of coverage

the cumulative mean of sparsity throughout the consecutive damage events and recovery.

Benchmark Movement Algorithms, The proposed movement algorithms are compared with several previouslypublished movement algorithms: distributed self spreading algorithm (DSSA)[10], which is a force-based movement algorithm), and Vor-Voronoi and MinMax-Voronoi, both based on Voronoi diagrams of the network [4], [7]. Limited version of Vor-Voronoi and MinMax-Voronoi, denoted Vor-Voronoi $(L)$ and MinMax-Voronoi( $(L)$, in which the nodes' movements are restricted when they move beyond their own range disc, are also compared with the proposed movement algorithm.

\section{RESUlts}

Using Matlab, $N=1000$ nodes with communication ranges of $15 \mathrm{~m}\left(R_{c}=R_{s}=15 \mathrm{~m}\right)$ are deployed at uniformly-distributed random locations in a rectangular deployment area of $[-100,100] \times[-100,100] \mathrm{m}$. Each coverage hole is modelled as a circle with a random radius $r_{\text {Hole }}, U_{r} \sim[0,50 \mathrm{~m}]$ and uniformly distributed random

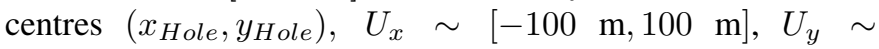
[-100 m, $100 \mathrm{~m}]$.

To properly compare the proposed movement algorithms with the benchmarks, we use randomly generated $\mathrm{CH}$ radii of $r_{\text {Hole }}^{\{1,2, \cdots, 5\}}=[47.3,48.9,35.2,41.8,42.5] \mathrm{m}$ and $\mathrm{CH}$ locations of $x_{\text {Hole }}^{\{1,2, \cdots, 5\}}=[23.5,-59,41.7,12.7,-67.3] \mathrm{m}$ and $y_{\text {Hole }}^{\{1,2, \cdots, 5\}}=[47,31,76.2,-18.5,-42] \mathrm{m}$ to model five consecutivley-formed coverage holes.
TABLE I

SPARSITY OF NETWORK WITH SINK NODES LOCATED AT $(100,100)$ M; 5 CONSECUTIVE CHS

\begin{tabular}{|l|lllll|}
\hline Algs. & \multicolumn{5}{|c|}{ Sparsity } \\
\hline & 1st & 2nd & 3rd & 4th & 5th \\
\hline \multirow{2}{*}{ SSM } & 1.7125 & 1.4266 & 1.2806 & 1.1905 & 1.1182 \\
& 1.7120 & 1.4410 & 1.2974 & 1.2049 & 1.1336 \\
\hline \multirow{2}{*}{ CSSCMM } & 1.8860 & 1.4527 & 1.2880 & 1.1550 & 1.0466 \\
& 1.7082 & 1.3169 & 1.1675 & 1.0471 & 0.9495 \\
\hline M- & 1.8969 & 1.4754 & 1.3304 & 1.1994 & 1.0973 \\
CSSCMM & 1.7995 & 1.4137 & 1.2814 & 1.1572 & 1.0611 \\
\hline aM- & 1.8897 & 1.4673 & 1.3223 & 1.1915 & 1.0893 \\
CSSCMM & 1.7831 & 1.3969 & 1.2653 & 1.1417 & 1.0460 \\
\hline dM- & 1.8201 & 1.4368 & 1.3084 & 1.1829 & 1.0824 \\
CSSMM & 1.6980 & 1.3584 & 1.2463 & 1.1290 & 1.0364 \\
\hline DSSA & 1.8035 & 1.4604 & 1.3845 & 1.2655 & 1.1686 \\
& 1.8324 & 1.4799 & 1.4012 & 1.2803 & 1.1816 \\
\hline \hline Vor- & 1.7352 & 1.4247 & 1.3859 & 1.2862 & 1.1972 \\
Voronoi & 2.0631 & 1.6582 & 1.5712 & 1.4493 & 1.3359 \\
\hline Vor- & 1.8448 & 1.4733 & 1.4025 & 1.2761 & 1.1767 \\
Voronoi(L) & 2.1112 & 1.6597 & 1.5399 & 1.3968 & 1.2816 \\
\hline \hline MinMax- & 1.7053 & 1.4147 & 1.3902 & 1.2842 & 1.1880 \\
Voronoi & 1.9868 & 1.5892 & 1.5042 & 1.3844 & 1.2706 \\
\hline MinMax- & 1.7275 & 1.4455 & 1.4056 & 1.2982 & 1.1994 \\
Voronoi(L) & 1.9957 & 1.6054 & 1.5191 & 1.4026 & 1.2865 \\
\hline
\end{tabular}

The performance of movement algorithms in terms of sparsity, percentage of coverage and node-to-sink distances has been evaluated for different algorithm parameters of $\lambda_{s}=$ $\{0.9,0.5,0.1\}\left(\lambda_{c m}=1-\lambda_{s}\right), \delta_{\tau}=0$ and sink node at the locations of $( \pm 100 \mathrm{~m}, \pm 100 \mathrm{~m})$. Due to similar performance trends for the given parameters, for sake of brevity, only the results of $\lambda_{s}=0.9$ with the sink node at the location of $(100 \mathrm{~m}, 100 \mathrm{~m})$ are presented in this paper. The experiment was repeated 50 times for all movement algorithms.

Performance of movement algorithms are shown in terms of networks sparsity (Table I and Fig. 5), percentage of coverage (Table II, Fig. 6), and boundary node to sink node distances (Table III, Fig. 7, 8, and 9). For comparison, in Tables I, II, and III, performance are calculated based on the efficacy of each algorithm applied after each occurrence of the damage events (i.e., the 2 nd row from each movement algorithms in the tables are used for comparison).

From Table I and Fig. 5, it can be seen that Voronoi-based movement algorithms and DSSA respectively outperform our proposed algorithms by $20 \%$ and $10 \%$. Among the proposed movement algorithms, only SSM improves network sparsity cut. Vor-Voronoi movement algorithms have the best performance, while DSSA outperforms SSM by about $5 \%$.

Results from Table II and Fig. 6 show that the proposed movement algorithms either slightly outperform or match DSSA. Voronoi-based algorithms outperform the proposed movement algorithms and DSSA with average of $20 \%-24 \%$ during recovery of consecutive CHs.

From Table III and Fig. 7, it can be seen that proposed movement algorithms slightly outperforms and/or matches Voronoi-based algorithms in terms of average boundary to sink node distance during the course of recovery process. DSSA 


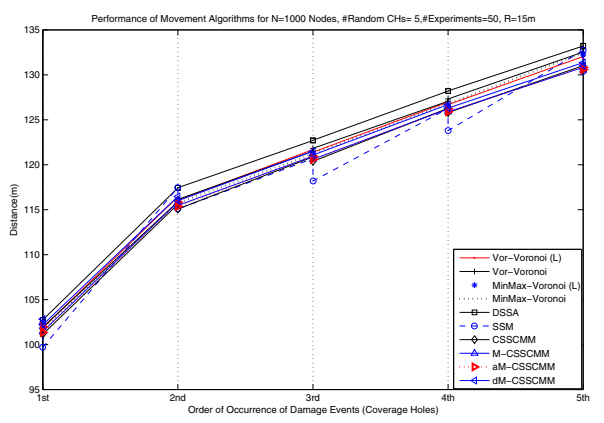

Fig. 7. Mean B-node to sink node distance

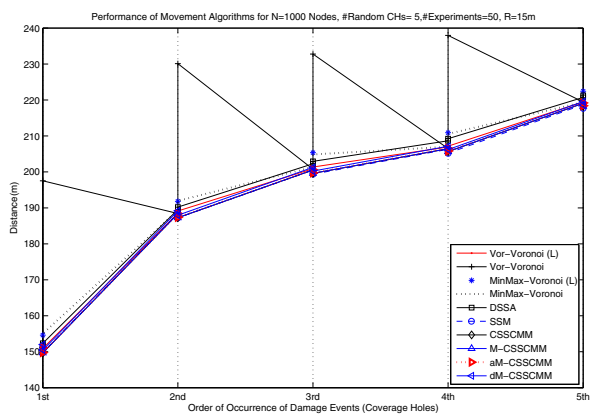

Fig. 8. Maximum B-node to sink node distance

has the worst performance among the other algorithms; SSM marginally has the best performance.

From Table III and Fig. 8, regarding the maximum distance from boundary to sink nodes, the proposed algorithms marginally outperform the DSSA and Voronoibased algorithms. SSM and Vor-Voronoi have the best and worst performances, respectively, as SSM outperforms Voronoi-based algorithms by an average of $17 \%$ in the course of recovery from consecutive coverage holes. The performance advantage for SSM decreases from $25 \%$ to $10 \%$ as number of $\mathrm{CHs}$ increase from 1 to 5. From Table III and Fig. 9, among all movement algorithms, the performance of SSM's maximumdistance boundary node to sink node is noteable; it varies from $15 \%$ to $40 \%$ in excess of the MinMax-Voronoi algorithm. SSM

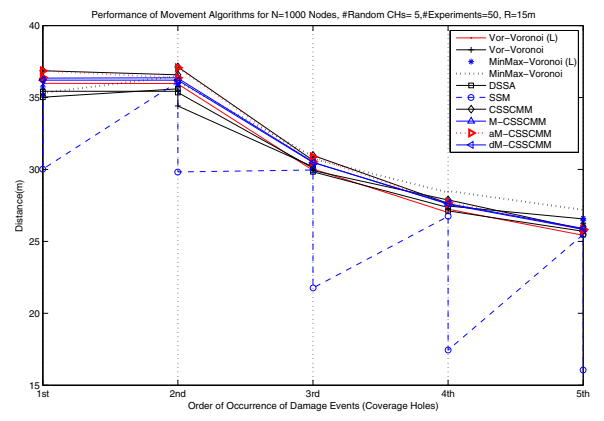

Fig. 9. Minimum B-node to sink node distances
TABLE II

Percentage of COVERAGE OF NETWORK, WITH SINK NODES LOCATED AT $(100,100)$ M; 5 CONSECUTIVE CHS

\begin{tabular}{|l|l|lllll|}
\hline Algs. & \multicolumn{5}{|c|}{ Percentage of Coverage(\%) } \\
\hline & Initial & 1 1st & 2nd & 3rd & 4th & 5th \\
\hline \multirow{2}{*}{ SSM } & 100 & 90.62 & 78.45 & 76.27 & 66.94 & 57.85 \\
& & 90.65 & 78.46 & 76.19 & 67.08 & 58.15 \\
\hline \multirow{2}{*}{ CSSCMM } & \multirow{2}{*}{100} & 90.65 & 78.59 & 76.08 & 67.46 & 58.35 \\
& & 90.82 & 78.81 & 76.32 & 67.62 & 58.42 \\
\hline M- & \multirow{2}{*}{100} & 90.63 & 78.57 & 75.88 & 66.92 & 57.64 \\
CSSCMM & & 90.79 & 78.68 & 75.97 & 66.99 & 57.68 \\
\hline aM- & \multirow{2}{*}{100} & 90.63 & 78.55 & 75.86 & 66.89 & 57.55 \\
CSSCMM & & 90.77 & 78.64 & 75.94 & 66.96 & 57.52 \\
\hline dM- & \multirow{2}{*}{100} & 90.56 & 78.63 & 76.02 & 66.93 & 57.93 \\
CSSMM & & 90.74 & 78.77 & 76.13 & 67.03 & 57.99 \\
\hline \multirow{2}{*}{ DSSA } & \multirow{2}{*}{100} & 90.55 & 78.47 & 75.88 & 67.31 & 58.52 \\
& & 90.68 & 78.70 & 76.17 & 67.48 & 58.70 \\
\hline \hline Vor- & \multirow{2}{*}{100} & 90.80 & 87.92 & 91.54 & 90.19 & 87.60 \\
Voronoi & & 99.65 & 97.31 & 98.24 & 97.00 & 96.00 \\
\hline Vor- & \multirow{2}{*}{100} & 90.62 & 86.00 & 89.09 & 87.62 & 86.01 \\
Voronoi(L) & & 97.15 & 94.64 & 95.94 & 95.33 & 93.10 \\
\hline \hline MinMax- & \multirow{2}{*}{100} & 90.63 & 87.36 & 91.28 & 90.11 & 89.27 \\
Voronoi & & 98.76 & 96.96 & 97.66 & 97.83 & 96.38 \\
\hline MinMax- & \multirow{2}{*}{100} & 90.63 & 87.33 & 91.28 & 90.22 & 88.83 \\
Voronoi(L) & & 98.76 & 96.89 & 97.75 & 97.53 & 96.16 \\
\hline
\end{tabular}

outperforms Voronoi-based algorithms by an average of $25 \%$. Such performance differences increase with occurrence of more CHs. Other proposed movement algorithms marginally outperform DSSA as well as Voronoi-based algorithms by $1 \%$ to $5 \%$.

\section{CONCLUSION}

This work present a new approach to the problem of recovery from coverage holes in WSNs via node autonomous node relocation algorithms. In the proposed recovery models, the the locations of deployed network sink nodes are modify the movement of nodes during the recovery process in order to conserve energy and maintain overall useable network longevity. The proposed distributed node relocation algorithms aim to reduce the moving nodes' distances to the sink nodes. This approach can be considered as an initial effort to partially address the gradual and impact of the sink nodes on the energy budget of their neighbours.

The performance of the proposed movement algorithms used in the coverage hole recovery model have been compared with previously published benchmark algorithms such as force-based and Voronoi-based relocation algorithms in terms of percentage of coverage, boundary nodes to sink distances and sparsity cut during the course of recovery from the consecutive random and large scale coverage holes in the network. The results show that there are trade-offs between targeted design goals of each relocation algorithm. Therefore, depending on the performance metric, the proposed model either outperforms, underperforms, or matches comparable algorithms. 
TABLE III

DistanCES TO SINK NODES LOCATED AT $(100,100)$ M; 5 CONSECUTIVE CHS

\begin{tabular}{|c|c|c|c|c|c|c|c|c|c|c|c|c|c|c|c|}
\hline \multirow[t]{2}{*}{ Algs. } & \multicolumn{5}{|c|}{$\begin{array}{l}\text { Average Distances } \\
\end{array}$} & \multicolumn{5}{|c|}{ Maximum Distances } & \multicolumn{5}{|c|}{$\begin{array}{l}\text { Minimum Distances } \\
\end{array}$} \\
\hline & 1 st & 2nd & 3rd & 4th & 5 th & 1 st & 2nd & 3rd & 4th & 5th & 1 st & 2nd & $3 \mathrm{rd}$ & 4 th & 5th \\
\hline & 102.35 & 117.47 & 120.69 & 126.25 & 132.73 & 151.30 & 188.47 & 200.53 & 206.31 & 218.73 & 36.25 & 36.09 & 29.96 & 26.74 & 25.48 \\
\hline SSM & 99.69 & 115.07 & 118.19 & 123.79 & 130.39 & 149.81 & 187.23 & 199.37 & 205.19 & 217.66 & 30.02 & 29.83 & 21.75 & 17.44 & 16.07 \\
\hline & 101.83 & 115.60 & 120.87 & 126.28 & 131.03 & 151.14 & 188.40 & 200.55 & 206.47 & 219.10 & 36.30 & 36.58 & 30.63 & 27.65 & 25.84 \\
\hline CSSCMM & 101.15 & 115.07 & 120.38 & 125.80 & 130.59 & 149.69 & 187.26 & 199.59 & 205.64 & 218.26 & 36.86 & 37.12 & 30.98 & 27.89 & 26.02 \\
\hline M- & 101.98 & 115.86 & 120.95 & 126.21 & 130.84 & 151.12 & 188.40 & 200.60 & 206.56 & 219.19 & 36.39 & 36.37 & 30.52 & 27.59 & 25.84 \\
\hline CSSCMM & 101.46 & 115.47 & 120.61 & 125.88 & 130.53 & 149.75 & 187.37 & 199.73 & 205.81 & 218.41 & 36.35 & 36.33 & 30.49 & 27.57 & 25.82 \\
\hline $\mathrm{aM}-$ & 101.94 & 115.81 & 121.01 & 126.30 & 130.96 & 151.13 & 188.41 & 200.61 & 206.57 & 219.16 & 36.39 & 36.62 & 30.69 & 27.64 & 25.79 \\
\hline CSSCMM & 101.35 & 115.36 & 120.59 & 125.89 & 130.58 & 149.75 & 187.38 & 199.74 & 205.81 & 218.39 & 36.85 & 37.06 & 30.87 & 27.71 & 25.80 \\
\hline $\mathrm{dM}-$ & 102.83 & 116.46 & 121.50 & 126.69 & 131.36 & 151.98 & 189.17 & 201.26 & 207.14 & 219.56 & 36.21 & 36.21 & 30.49 & 27.62 & 25.90 \\
\hline CSSMM & 102.21 & 115.99 & 121.08 & 126.29 & 130.99 & 150.49 & 187.97 & 200.23 & 206.21 & 218.61 & 36.21 & 36.21 & 30.49 & 27.62 & 25.89 \\
\hline & 102.73 & 117.42 & 122.67 & 128.19 & 133.21 & 151.80 & 189.53 & 202.27 & 208.65 & 220.71 & 35.41 & 35.60 & 30.10 & 27.35 & 25.70 \\
\hline DSSA & 102.75 & 117.45 & 122.72 & 128.21 & 133.23 & 152.35 & 190.22 & 202.93 & 209.23 & 221.29 & 35.02 & 29.84 & 17.52 & 27.12 & 25.48 \\
\hline Vor- & 102.31 & 115.87 & 121.51 & 127.06 & 132.54 & 150.49 & 188.43 & 200.74 & 206.51 & 219.44 & 35.44 & 35.44 & 30.19 & 27.88 & 26.56 \\
\hline Voronoi & 101.94 & 116.14 & 121.83 & 127.32 & 132.89 & 197.52 & 230.11 & 232.78 & 237.96 & 244.44 & 35.44 & 34.41 & 29.92 & 27.46 & 26.25 \\
\hline Vor- & 102.59 & 116.44 & 121.68 & 126.98 & 132.03 & 150.95 & 188.50 & 200.88 & 206.93 & 219.46 & 35.96 & 35.97 & 29.94 & 27.01 & 25.42 \\
\hline Voronoi(L) & 101.85 & 116.06 & 121.40 & 126.68 & 131.79 & 150.93 & 189.13 & 201.35 & 207.16 & 219.47 & 35.98 & 35.94 & 30.03 & 27.24 & 25.64 \\
\hline "MinMax- & 102.32 & 115.81 & 121.19 & 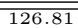 & $\overline{132.49}$ & 151.85 & 189.34 & 201.35 & $\overline{206.99}$ & $\overline{219.40}$ & $\overline{36.21}$ & 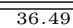 & 30.90 & 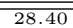 & $\overline{27.19}$ \\
\hline Voronoi & 101.76 & 115.76 & 121.25 & 126.79 & 132.45 & 155.05 & 191.96 & 204.87 & 210.57 & 222.32 & 35.32 & 36.11 & 30.74 & 28.50 & 27.53 \\
\hline MinMax- & 102.55 & 189.02 & 120.98 & 126.51 & 132.20 & 151.59 & 226.44 & 201.23 & 206.91 & 219.28 & 35.74 & 35.96 & 30.33 & 27.70 & 26.44 \\
\hline Voronoi(L) & 101.88 & 115.95 & 121.12 & 126.59 & 132.31 & 154.61 & 191.83 & 205.35 & 210.90 & 222.44 & 35.13 & 35.80 & 30.15 & 27.78 & 26.61 \\
\hline
\end{tabular}

\section{REFERENCES}

[1] L. Paradis and Q. Han, "A survey of fault management in wireless sensor networks," J. Netw. Syst. Manage., vol. 15, no. 2, pp. 171-190, Jun. 2007.

[2] N. Ahmed, S. S. Kanhere, and S. Jha, "The holes problem in wireless sensor networks: a survey," SIGMOBILE Mob. Comput. Commun. Rev., vol. 9, no. 2, pp. 4-18, 2005.

[3] N. Jabeur, N. Sahli, and I. M. Khan, "Survey on sensor holes: A cause-effect-solution perspective," Procedia Computer Science, vol. 19, no. 0, pp. $1074-1080,2013$, the 4th International Conference on Ambient Systems, Networks and Technologies (ANT 2013), the 3rd International Conference on Sustainable Energy Information Technology (SEIT-2013).

[4] A. Rafiei, M. Abolhasan, D. Franklin, and F. Safaei, "Wsns coverage hole partial recovery by nodes' constrained and autonomous movements using virtual alpha-chords," in ICWMC 2012, The Eighth International Conference on Wireless and Mobile Communications, 2012, pp. 74-80.

[5] R. R. Roy, Handbook of mobile ad hoc networks for mobility models. New York, NY: Springer, 2011.

[6] B. Wang, Coverage Control in Sensor Networks, 1st ed. Springer Publishing Company, Incorporated, 2010.

[7] M. Argany, M. Mostafavi, and F. Karimipour, "Voronoi-based approaches for geosensor networks coverage determination and optimisation: A survey," in 2010 International Symposium on Voronoi Diagrams in Science and Engineering (ISVD), June 2010, pp. 115-123.

[8] A. Sekhar, B. Manoj, and C. Murthy, "Dynamic coverage maintenance algorithms for sensor networks with limited mobility," in Third IEEE International Conference on Pervasive Computing and Communications, PerCom 2005, March 2005, pp. 51-60.

[9] S. Chellappan, W. Gu, X. Bai, D. Xuan, B. Ma, and K. Zhang, "Deploying wireless sensor networks under limited mobility constraints," Mobile Computing, IEEE Transactions on, vol. 6, no. 10 , pp. $1142-1157$, oct. 2007.

[10] N. Heo and P. Varshney, "A distributed self spreading algorithm for mobile wireless sensor networks," in Wireless Communications and Networking, 2003. WCNC 2003. 2003 IEEE, vol. 3, March 2003, pp. $1597-1602$ vol.3.

[11] M. Labrador and P. Wightman, Topology Control in Wireless Sensor Networks: with a companion simulation tool for teaching and research. Springer, 2009.

[12] H. Coxeter, "Inversion in a circle and inversion of lines and circles," Introduction to Geometry, 2nd ed. John Wiley and Sons, New York, pp. 77-83, 1969.
[13] J. Yick, B. Mukherjee, and D. Ghosal, "Wireless sensor network survey," Computer Networks, vol. 52, no. 12, pp. 2292 - 2330, 2008.

[14] M. Burgess, "An approach to understanding policy based on autonomy and voluntary cooperation," in Ambient Networks, ser. Lecture Notes in Computer Science, J. Schnwlder and J. Serrat, Eds. Springer Berlin Heidelberg, 2005, vol. 3775, pp. 97-108.

[15] A. Aziz, Y. Sekercioglu, P. Fitzpatrick, and M. Ivanovich, "A survey on distributed topology control techniques for extending the lifetime of battery powered wireless sensor networks," Communications Surveys Tutorials, IEEE, vol. PP, no. 99, pp. 1 -24, 2012.

[16] A. Casteigts, J. Albert, S. Chaumette, A. Nayak, and I. Stojmenovic and, "Biconnecting a network of mobile robots using virtual angular forces," in Vehicular Technology Conference Fall (VTC 2010-Fall), 2010 IEEE 72nd, Sept. 2010, pp. 1 -5.

[17] A. Rafiei, Y. Maali, M. Abolhasan, D. R. Franklin, F. Safaei, and S. Smith, "A tuned fuzzy logic relocation model in wsn using particle swarm optimization," in 2013 IEEE 78th Vehicular Technology Conference: VTC2013-Fall 2-5 September 2013, Las Vegas, USA, September 2013.

[18] A. Rafiei, M. Abolhasan, D. R. Franklin, and S. Smith, "A case study for choosing proper relocation algorithms to recover large scale coverage hole(s) in wireless sensor networks," in 1st Workshop on Advances in Real-time Information Networks, 7-10 August, Sydney, Australia. epress UTS publishing, August 2013.

[19] I. Amundson and X. D. Koutsoukos, "A survey on localization for mobile wireless sensor networks," in Proceedings of the 2nd international conference on Mobile entity localization and tracking in GPS-less environments, ser. MELT'09. Berlin, Heidelberg: Springer-Verlag, 2009, pp. 235-254.

[20] R. Luce and A. Perry, "A method of matrix analysis of group structure," Psychometrika, vol. 14, no. 2, pp. 95-116, 1949. [Online]. Available: http://dx.doi.org/10.1007/BF02289146

[21] L. Trevisan, "CS359G: Graph Partitioning and Expanders, Lecture 1," Course at Stanford University, http://www.eecs.berkeley.edu/[̃Aluca/cs359g/index.html, January 2011.

[22] M. M. Deza and M. Laurent, Geometry of Cuts and Metrics. Springer Berlin Heidelberg, 1997. [Online]. Available: http://dx.doi.org/10.1007/978-3-642-04295-9 